\title{
Synthesis and Characterization \\ of Lead Dibutyl Dithiophosphate Nanoparticles
}

\author{
Svetlana V. Saikova ${ }^{a, b}$, \\ Dmitriy I. Chistyakova, Diana I. Saykova ${ }^{a}$, \\ Yury L. Mikhlin ${ }^{b}$ and Dmitriy V. Kuzmin ${ }^{b}$ \\ ${ }^{a}$ Siberian Federal University \\ Krasnoyarsk, Russian Federation \\ ${ }^{b}$ Institute of Chemistry and Chemical Technology SB RAS \\ Krasnoyarsk, Russian Federation
}

Received 08.05.2021, received in revised form 30.07.2021, accepted 16.08.2021

\begin{abstract}
We examined the interaction of aqueous solutions of lead nitrate and sodium dibutyl dithiophosphate and showed the formation nanoparticles of lead dibutyl dithiophosphate. The effect of reaction parameters on the synthesis of nanoparticles was studied and the optimal conditions for the formation of their stable hydrosols were determined. The obtained products were investigated by methods of optical spectroscopy, dynamic light scattering. The average size of $15-20 \mathrm{~nm}$, but due to the low surface charge, they are combined into larger aggregates. The UV-vis spectra has absorption maximum at about $320 \mathrm{~nm}$. TEM micrographs and $\mathrm{Pb} 2 \mathrm{p}, \mathrm{S} 2 \mathrm{p}$ XAS spectra revealed a composition and structure of the particles. These nanoparticles can play an important role in the flotation of sulfide minerals of nonferrous metals.
\end{abstract}

Keywords: nanoparticles, flotation, aeroflots, dibutyl dithiophosphates, optical spectroscopy, dynamic light scattering, X-ray photoelectron spectroscopy.

Citation: Saikova, S. V., Chistyakov, D. I., Saykova, D. I., Mikhlin, Yu. L., Kuzmin, D. V. Synthesis and characterization of lead dibutyl dithiophosphate nanoparticles, J. Sib. Fed. Univ. Chem., 2021, 14(3), 350-359. DOI: 10.17516/1998-2836-0243

(C) Siberian Federal University. All rights reserved

This work is licensed under a Creative Commons Attribution-NonCommercial 4.0 International License (CC BY-NC 4.0).

* Corresponding author E-mail address: ssai@mail.ru 


\title{
Синтез и характеризация наночастиц дибутилдитиофосфата свинца
}

\author{
С. В. Сайкова ${ }^{a, ~ б, ~ Д . ~ И . ~ Ч и с т я к о в ~}{ }^{a}$, \\ Д. И. Сайкова ${ }^{a}$, Ю. Л. Михлин ${ }^{\boldsymbol{0}}$, Д. В. Кузьмин \\ ${ }^{a}$ Сибирский федеральньий университет \\ Российская Федераџия, Красноярск \\ ${ }^{6}$ Институт химии и химической технологии СО РАН \\ ФИЦ «Красноярский научный центр СО РАН» \\ Российская Федераџия, Красноярск
}

\begin{abstract}
Аннотация. Показано, что при взаимодействии водных растворов нитрата свинца и дибутилдитиофосфата натрия формируются гидрозоли, содержащие наночастицы дибутилдитиофосфата свинца (ДБДТФ $\mathrm{Pb}$ ), которые могут играть важную роль в процессах флотации сульфидных минералов цветных металлов. Изучено влияние реакционных параметров на синтез наночастиц и определены оптимальные условия формирования их устойчивых гидрозолей. Исследование полученных продуктов методами оптической спектроскопии, динамического рассеяния света, рентгеновской фотоэлектронной спектроскопии и просвечивающей электронной микроскопии показало, что наночастицы ДБДТФ Рb имеют средний размер 15-20 нм, но вследствие низкого заряда поверхности объединяются в более крупные агрегаты.
\end{abstract}

Ключевые слова: наночастицы, флотация, аэрофлоты, дибутилдитиофосфаты, оптическая спектроскопия, динамическое рассеяние света, рентгеновская фотоэлектронная спектроскопия.

Цитирование: Сайкова, С. В. Синтез и характеризация наночастиц дибутилдитиофосфата свинца / С. В. Сайкова, Д. И. Чистяков, Д. И. Сайкова, Ю. Л. Михлин, Д. В. Кузьмин // Журн. Сиб. федер. ун-та. Химия, 2021, 14(3). С. 350-359. DOI: 10.17516/1998-2836-0243

\section{Введение}

Диалкилдитиофосфаты (аэрофлоты) используются в качестве эффективных собирателей при флотации сульфидных медных минералов (халькозин, ковеллин, халькопирит, борнит), цинковых минералов (сфалерит, марматит), активированных ионами меди, драгоценных и благородных металлов (Au, Ag, Pt, Os, и т. п.), а также их минералов (аргентит, калазерит, сильванит и т.п). [1]. При взаимодействии аэрофлотов с тонкими классами сульфидных минералов образуются наноразмерные частицы (нч), которые значительно улучшают их флотацию. Кроме того, дитиофосфаты проявляют селективные свойства при отделении сульфидов цветных металлов от сульфидов железа (пирит, пирротин, арсенопирит), а также эффективны при разделении коллективных концентратов, поскольку легче, чем ксантогенат, десорбируются с поверхности минералов [2-4]. Многие авторы объясняют положительное влияние аэрофлотов на эффективность флотации особой устойчивостью их соединений с тяжелыми металлами, зачастую имеющих структуру хелатов [5-7]. Считается, что дитиофосфаты металлов образуют 
наноразмерные частицы, которые формируются либо на поверхности минерала, либо в раствоpe, поскольку при измельчении флотируемые сульфидные минералы поверхностно окисляются и ионы металла переходят в раствор [3]. Образующиеся в растворе наночастицы способны адсорбироваться на поверхности минерала, что оказывает положительное влияние на процесс флотации [8-11].

По зарубежным данным, доля аэрофлотов в общем объеме потребления сульфгидрильных собирателей составляет около 25 \%. При этом на российских предприятиях эти флотореагенты находят весьма ограниченное применение. Между тем имеющийся опыт доказывает их эффективность и экономическую целесообразность $[12,13]$.

Диалкилдитиофосфаты являются солями вторичных кислых сложных эфиров дитиофосфорной кислоты и какого-либо спирта. В их молекулах один атом серы двойной связью присоединен к фосфору, а другой связан с фосфором и водородом. Дитиофосфаты щелочных металлов, будучи ионными соединениями, хорошо растворимы в воде, спиртах, низших кетонах, пиридине, ацетонитриле и практически не растворяются в неполярных растворителях. Диалкилдитиофосфаты цветных металлов исследованы в недостаточной степени, а имеющиеся данные в основном относятся к продуктам, полученным в неводных растворителях, или твердым соединениям. В литературе практически отсутствуют результаты, касающиеся получения и исследования наночастиц диалкилдитиофосфатов. В нашей предыдущей работе [14] получены и исследованы наночастицы дибутилдитиофосфата меди. Цели данной работы - разработка методики синтеза наноразмерных частиц дибутилдитиофосфата свинца при взаимодействии исходных растворов дибутилдитиофосфата натрия и $\mathrm{Pb}\left(\mathrm{NO}_{3}\right)_{2}$ и изучение полученных наночастиц методами оптической спектроскопии, просвечивающей электронной микроскопии (ПЭМ), рентгенофотоэлектронной спектроскопии (РФЭС) и фотонно-корреляционной спектроскопии (ФКС).

\section{Материалы и методы}

В работе применяли дибутилдитиофосфат натрия (ДБДТФН) квалификации «техн.» и нитрат свинца «ч. Д. а.». ДБДТФН предварительно очищен и исследован на содержание общего и органического фосфора [15]. По результатам анализа, чистота ДБДТФН составила 98 \%.

К 10 мл раствора ДБДТФН, концентрацию которого варьировали в интервале от 2 до 8 мМ, прибавляли такой же объем раствора $\mathrm{Pb}\left(\mathrm{NO}_{3}\right)_{2}$ с концентрацией 2 мМ. Предварительно все растворы нагревали в течение 20 мин на водяной бане до заданной температуры $\left(20-60{ }^{\circ} \mathrm{C}\right)$. В ходе реакции образовывался гидрозоль белого цвета, устойчивый при любых использованных концентрациях и температурах.

Образовавшиеся гидрозоли изучали спектрофотометрически (Specol 1300) в диапазоне длин волн 200-800 нм в кварцевой кювете с длиной оптического слоя 1 см. При необходимости перед измерением образцы разбавляли дистиллированной водой.

При исследовании методом рентгеновской фотоэлектронной спектроскопии каплю полученного золя высушивали на пирографитовой подложке в вакууме шлюзовой камеры спектрометра SPECS, снабженного полусферическим анализатором электронов PHOIBOS150-MCD-9. Спектры образцов записывали при возбуждении монохроматизированным излучением $\mathrm{AlK}_{\alpha}$ (1486,6 эВ). Давление в аналитической камере было ниже $10^{-9}$ мБар. В качестве внутреннего 
стандарта для учета электростатической подзарядки использовалась линия C 1s BОПГ $(285,0$ эВ). Обработку спектров РФЭС выполняли с помощью программы CasaXPS с вычитанием фона по Ширли и Гаусс-Лоренцевым разложением линий. Травление ионами $\mathrm{Ar}^{+}$осуществляли со скоростью примерно 2 нм/мин (ускоряющее напряжение 5 кВ, ток эмиссии 30 мкА). С ростом энергии возбуждения растет длина свободного пробега фотоэлектрона и, как следствие, глубина анализируемого слоя. Использование для возбуждения линий $\mathrm{Mg} \mathrm{K \alpha}(1253,6$ эВ) и $\mathrm{Al}$ К $\alpha$ (1486,6 эВ) позволяет анализировать слой толщиной около 1,5 и 1,7 нм соответственно, т. е. с некоторым разрешением по глубине.

Для определения гидродинамического диаметра полученных частиц методом динамического светорассеяния использовали прибор Zetasizer Nano ZS (Malvern Instruments Ltd, Великобритания), оснащенный гелий-неоновым лазером (длина волны 633 нм, мощность 4 мBТ). Измерения проводили при угле рассеяния $173^{\circ}$ в термостатируемой ячейке при необходимой температуре с использованием одноразовых 12 мм полистирольных кювет. Размер частиц определяли как среднее из трех измерений. В качестве выходного параметра использовался средний объемный размер.

Исследование методом ПЭМ осуществляли на приборе JEM-2100 (JEOL) при ускоряющем напряжении 200 кВ. Золь перед исследованием диспергировали в УЗ-ванне. Каплю полученного золя наносили на медную сетку, покрытую аморфной углеродной пленкой, и высушивали на воздухе.

\section{Результаты и обсуждение}

Синтез наночастиц дибутилдитиофосфата свинца проводили сливанием равных объемов водных растворов нитрата свинца и дибутилдитиофосфата натрия. Было установлено, что взаимодействие не сопровождается окислительно-восстановительной реакцией и протекает в соответствии с уравнением

$$
\mathrm{Pb}\left(\mathrm{NO}_{3}\right)_{2}+2\left(\mathrm{C}_{4} \mathrm{H}_{9} \mathrm{O}\right)_{2} \mathrm{PSSNa}=\mathrm{Pb}\left(\left(\mathrm{C}_{4} \mathrm{H}_{9} \mathrm{O}\right)_{2} \mathrm{PSS}\right)_{2}+2 \mathrm{NaNO}_{3} .
$$

Стехиометрическим является молярное отношение ионов $\mathrm{Pb}^{2+}$ и ДБДТФН, равное 0,5. Все полученные золи были седиментационно стабильны, формирования осадка не наблюдалось.

На рисунке 1 представлены электронные спектры поглощения (ЭСП) гидрозолей, полученных при различных концентрациях ДБДТФН (от 2 до 8 ммоль/л), после их 15-кратного разбавления. В ЭСП наблюдается небольшой максимум при 320 нм. Сам спектр наложен на фон, связанный со светорассеянием на частицах коллоидного размера (теория светорассеяния Ми [16]).

Известно [17], что в спектре раствора дибутилдитиофосфата свинца в $\mathrm{CCl}_{4}$ наблюдается максимум поглощения вблизи 300 нм. При образовании наночастиц ДБДТФ свинца в водном растворе максимум смещается к 320 нм. Согласно полученным данным, концентрация наночастиц дибутилдитиофосфата свинца в дисперсиях не подчиняется закону Бугера-ЛамбертаБера, т. е. линейной зависимости величины поглощения (на 320 нм) от концентрации нч не наблюдается. Однако в работах $[18,19]$ на примере коллоидов различных материалов, таких как $\mathrm{MoS}_{2}, \mathrm{WS}_{2}$, BN, сообщалось о соблюдении данной закономерности в довольно широком интервале концентраций дисперсий.

$$
-353-
$$


Увеличение интенсивности максимума поглощения при повышении концентрации аниона говорит о росте выхода наночастиц. В дальнейших экспериментах использовали концентрации ДБТДФ 4-8 мМ, что соответствовало 2-4-кратному его избытку над стехиометрией реакции (1).

На рисунке 2 приведены спектры гидрозоля дибутилдитиофосфата свинца, полученного при взаимодействии 8 мМ раствора ДБТДФ и 2 мМ нитрата свинца, они записаны спустя 15 (кривая 1) и 45 (кривая 2) мин от начала синтеза. Снижение интенсивности максимума поглощения со временем можно объяснить агрегацией частиц ДБДТФ свинца. Данное предположение подтверждается и фактом несоблюдения закона Бугера-Ламберта-Бера для ЭСП гидрозолей

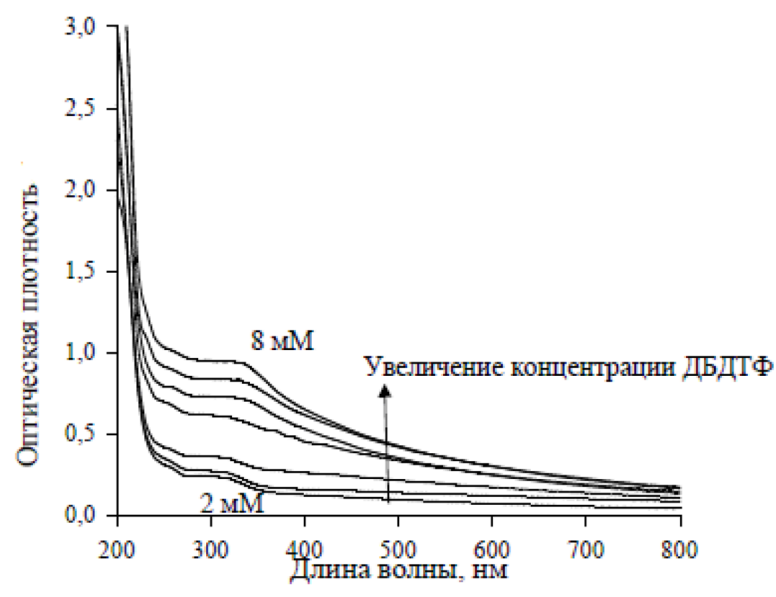

Рис. 1. Изменение вида оптических спектров поглощения золей дибутилдитиофосфата свинца с ростом концентрации ДБДТФН от 2 до 8 ммоль/л

Fig. 1. Effect of sodium dibutyldithiophosphate concentration $\left(2-8 \mathrm{mmol} \cdot \mathrm{L}^{-1}\right)$ on $\mathrm{UV}-\mathrm{Vis}$ absorption spectra of lead dibutyldithiophosphate sols

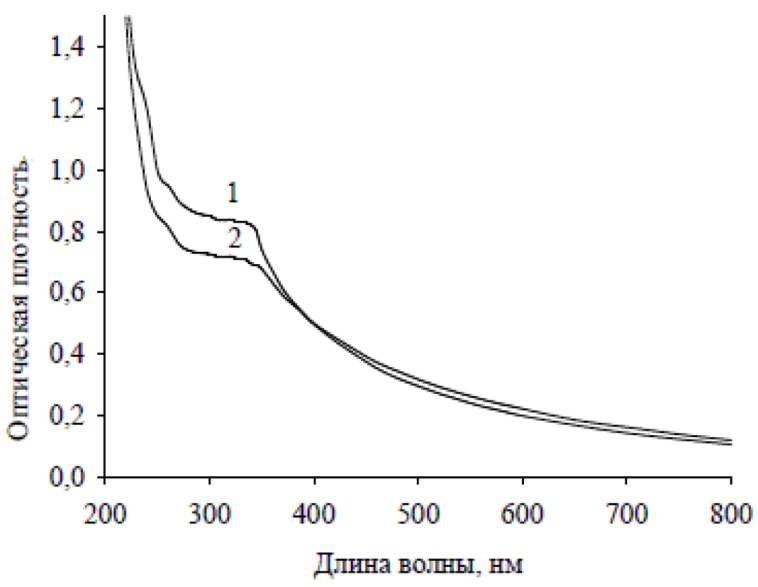

Рис. 2. Изменение вида оптических спектров поглощения золей дибутилдитиофосфата свинца (концентрация ДБДТФН 8 ммоль/л) со временем: 1-15 мин; 2-45 мин

Fig. 2. Time evolution of UV-Vis absorption spectra of lead dibutyldithiophosphate sols (sodium dibutyldithiophosphate concentration $\left.\mathrm{mmol} \cdot \mathrm{L}^{-1}\right): 1-15 \mathrm{~min} ; 2-45 \mathrm{~min}$ 
a
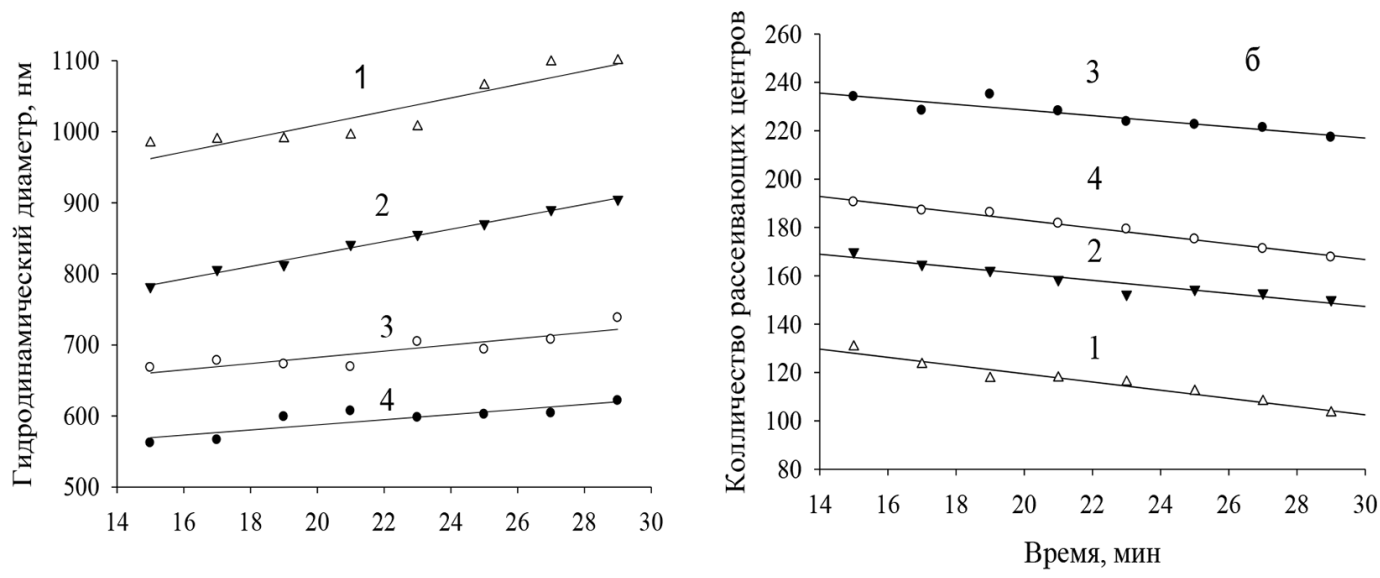

Рис. 3. Влияние температуры синтеза (1-60, 2-50, 3-40, 4-25 $\left.{ }^{\circ} \mathrm{C}\right)$ на динамику изменения гидродинамического диаметра частиц дибутилдитиофосфата свинца и количества рассеивающих центров

Fig. 3. Time evolution of hydrodynamic diameter of the lead dibutyldithiophosphate particles (a) and the number of scattering centers (в) at various temperatures, ${ }^{\circ} \mathrm{C}: 1-60,2-50,3-40,4-25$

наночастиц дибутилдитиофосфата свинца, а также результатами ФКС (рис. 3). С увеличением времени синтеза гидродинамический диаметр нч ДБДТФ свинца растет, причем этот рост сопровождается снижением количества рассеивающих центров (рис. 3б), т. е. вызван агрегацией наночастиц.

При увеличении температуры синтеза от 25 до $60{ }^{\circ} \mathrm{C}$ наблюдался заметный рост гидродинамического диаметра частиц (от 550 до 1100 нм), который также сопровождался снижением количества рассеивающих центров, что говорит о том, что агрегативная устойчивость полученных наночастиц снижается при повышении температуры (рис. 3).

Эффект разбавления золя, содержащего частицы дибутилдитиофосфата свинца (концентрация ДБДТФН $5 \cdot 10^{-3} \mathrm{M}$, температура синтеза $30^{\circ} \mathrm{C}$ ), на их гидродинамический диаметр и число рассеивающих центров представлен на рис. 4. При разбавлении золя в 15 раз гидродинамический диаметр его частиц и число рассеивающих центров закономерно снижаются.

По данным метода ФКС, полученные частицы дибутилдитиофосфата свинца имеют субмикронный размер, однако исследование методом просвечивающей электронной микроскопии показывает (рис. 5), что реальный размер частиц меньше их гидродинамического диаметра и равен 15-20 нм. При этом они объединяются и формируют глобулы размером 70-100 нм.

$\mathrm{C}$ целью объяснения причин образования агломератов измерили значение $\xi$-потенциала частиц ДБДТФ свинца. Согласно полученным данным, частицы в дисперсиях практически не заряжены (среднее значение -потенциала 0,41 мВ). Отсутствие поверхностного заряда, который мог бы обеспечить электростатическое отталкивание между частицами, объясняет агрегативную неустойчивость полученных лиофобных гидрозолей (нейтрализационная коагуляция).

Агломераты частиц могут быть разбиты на более мелкие части с помощью непродолжительной (в течение 5-8 мин) УЗ-обработки при температуре $60^{\circ} \mathrm{C}$. Гидродинамический диаметр 

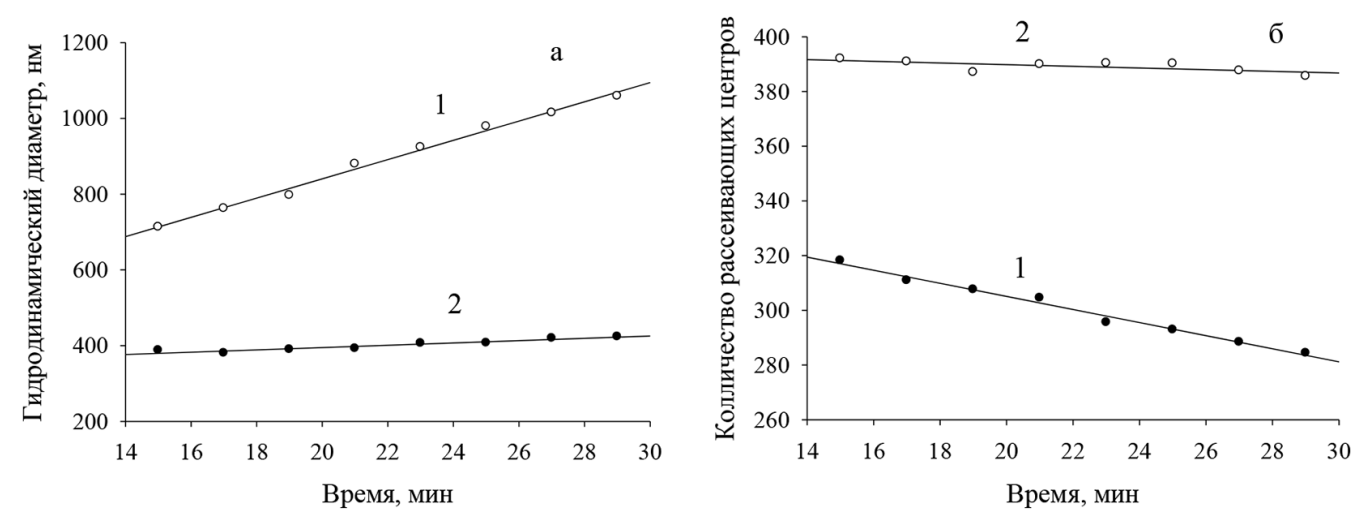

Рис. 4. Влияние разбавления (1 - без разбавления, 2-15-кратное разбавление) на динамику изменения гидродинамического диаметра частиц дибутилдитиофосфата свинца и количества рассеивающих центров

Fig. 4. Time evolution of hydrodynamic diameter of the lead dibutyldithiophosphate particles (a) and the number of scattering centers (в) with (2) and without (1) 15-fold dilution of the sols

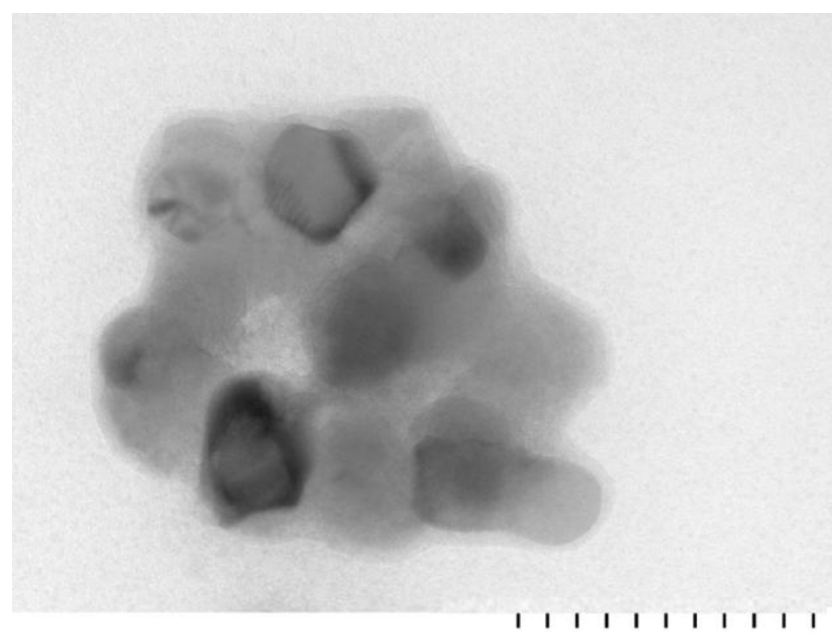

$50 \mathrm{~nm}$

Рис. 5. Микрофотография (ПЭМ) наночастиц дибутилдитиофосфата свинца

Fig. 5. Typical TEM micrographs of lead dibutyldithiophosphate nanoparticles

частиц при этом снижался до 200 нм. Однако воздействие ультразвуком имеет краткосрочный эффект. В течение 30 мин частицы вновь формируют агрегаты, а их гидродинамический диаметр возвращается к своим прежним размерам.

\section{Исследование нч дибутилдитиофосфата свинща методом РФЭС}

Методом РФЭС с разрешением по глубине было проведено изучение химического состояния поверхности полученных образцов нч дибутилдитиофосфата свинца. На основании анализа обзорных спектров установили, что в них отсутствуют линии, соответствующие натрию, что говорит о хорошей очистке образца от исходных реагентов. Элементный состав частиц ги- 


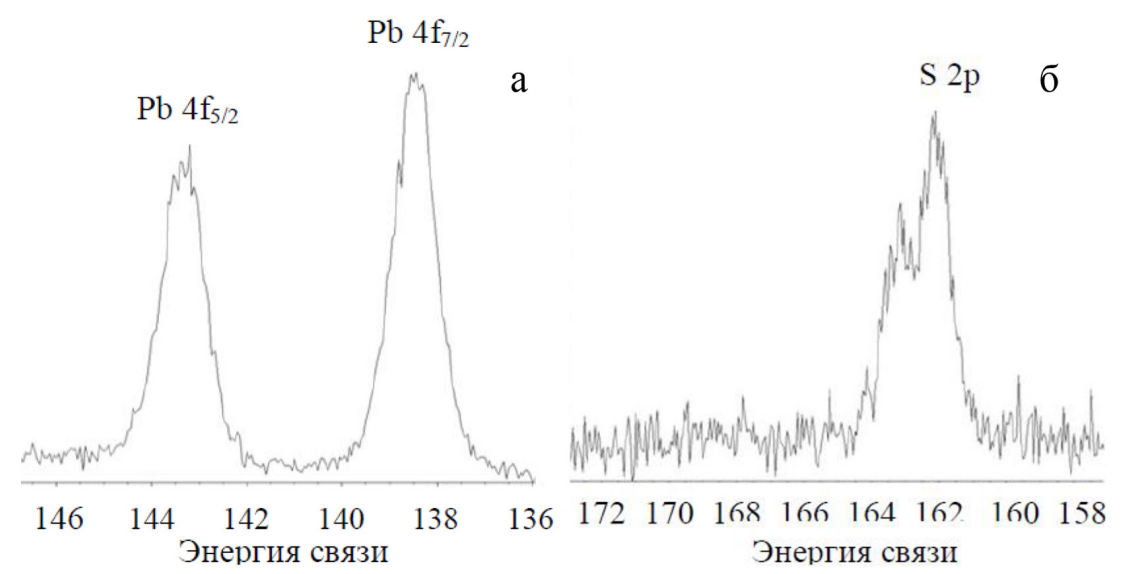

Рис. 6. Рентгенофотоэлектронные спектры образцов дибутилдитиофосфата свинца: $\mathrm{a}$ - линия $\mathrm{Pb} 4 \mathrm{f}, 6$ линия $\mathrm{S} 2 \mathrm{p}$

Fig. 6. X-ray photoelectronic spectra (a) $\mathrm{Pb} 4 \mathrm{f}$ and (b) S2p of lead dibutyldithiophosphate

дрозолей характерен для дибутилдитиофосфата свинца. На рис. 6 представлены узкие сканы. Линия S2p состоит из двух компонент: основной с энергией связи 162,4 эВ и второстепенной при 163,6 эВ, что характерно для исходного дибутилдитиофосфата натрия [14]. Остальные линии - Р, С, О (не приводятся) также соответствуют дибутилдитиофосфат-иону. Линия $\mathrm{Pb} 4 \mathrm{f}_{7 / 2}$ с энергией связи 138,4 эВ свидетельствует, что свинец имеет степень окисления +2 . Таким образом, данные РФЭС подтверждают состав полученного продукта $-\mathrm{Pb}\left(\left(\mathrm{C}_{4} \mathrm{H}_{9} \mathrm{O}\right)_{2} \mathrm{PSS}\right)_{2}$.

\section{Заключение}

В ходе исследования изучено влияние различных факторов на синтез гидрозолей дибутилдитиофосфата свинца и найдены оптимальные условия процесса: концентрации $\mathrm{Pb}\left(\mathrm{NO}_{3}\right)_{2}-0,4$ ммоль/л и ДБДТФ - 1 ммоль/л, время синтеза 15 мин, температура $30{ }^{\circ} \mathrm{C}$. Полученные гидрозоли имеют максимум поглощения при 320 нм, отвечающий формированию наночастиц дибутилдитиофосфата свинца. По результатам просвечивающей электронной микроскопии наночастицы ДБДТФ свинца имеют размер 15-20 нм, но объединяются с формированием глобул размером 70-100 нм. Гидродинамический диаметр, определенный методом ФКС, зависит от условий синтеза и варьирует в интервале 600-800 нм, что доказывает агрегирование синтезированных частиц вследствие низкого значения их $\zeta$-потенциала $(0,41 \mathrm{mB})$. При исследовании полученных частиц методом РФЭС подтверждено образование дибутилдитиофосфата свинца состава $\mathrm{Pb}\left(\left(\mathrm{C}_{4} \mathrm{H}_{9} \mathrm{O}\right)_{2} \mathrm{PSS}\right)_{2}$. Проведенное исследование процесса синтеза наночастиц дибутилдитиофосфата свинца подтверждает, что они могут образовываться в условиях флотации. Адсорбция таких частиц на поверхности минерала формирует микрорельеф, который увеличивает ее гидрофобность, что положительно сказывается на флотации.

\section{Список литературы / References}

1. Абрамов А.А. Флотационные методы обогащения. М.: Недра, 1993. 412 с. [Abramov A. A. Flotation methods of beneficiation. Moscow: Nedra, 1993. 412 p. (In Russ.)] 
2. Рябой В.И., Асончик К. М., Полькин В.Н., Полтавская Л. М., Репина Н. В. Применение селективного собирателя при флотации медно-цинковых руд. Обогащение руд. 2008. Т. 3, С. 20 22. [Ryaboy V.I., Asonchik K. M., Polkin V.N., Poltavskaya L.M., Repina N. V. The use of a selective collector in the flotation of copper-zinc ores. Obogashchenie rud. 2008. Vol. 3, P. 20-22. (In Russ.)]

3. Ignatkina V.A., Bocharov V.A., D'yachkov F.G. Collecting properties of diisobutyl dithiophosphinate in sulfide minerals flotation from sulfide ore. Journal of Mining Science. 2012. Vol. 49, P. 795-802.

4. Ignatkina V.A., Bocharov V.A., Kayumov A.A. Basic principles of selecting separation methods for sulfide minerals having similar properties in complex ore concentrates. Journal of Mining Science. 2016. Vol. 52, P. 360-372.

5. Nagaraj D.R., Farinato R.S. Evolution of flotation chemistry and chemicals: A century of innovations and the lingering challenges. Minerals Engineering. 2016. Vol. 96-97. P. 2-14.

6. Mahmoud M. R., Lazaridis N. K., Matis K. A. Study of flotation conditions for cadmium(II) removal from aqueous solutions. Process Safety and Environmental Protection. 2015. Vol. 94. P. $203-$ 211.

7. Rao S.R. Surface Chemistry of Froth Flotation Volume 1: Fundamentals Second Edition. New York, Springer Science+Business Media. 2004. P. 257-292.

8. Abdalla M. A. M., Peng H., Wu D., Abusin L., Mbah T. J. Prediction of Hydrophobic Reagent for Flotation Process Using Molecular Modeling. ACS Omega. 2018. Vol. 3(6). P. 6483-6496.

9. Mikhlin Y.L., Romanchenko A.S., Vorobyev S.A., Karacharov A.A., Likhatski M.N., Tomashevich Y.V., Saikova S. V. Insights into nanoscale phenomena on the sulfide mineral surfaces related to flotation and leaching. IMPC2018-29th International Mineral Processing Congress. P. 15421551.

10. Vorobyev S. A., Saikova S. V., Novikova S. A., Fetisova O. Y., Zharkov S. M., Krylov A.S., Likhatski M.N., Mikhlin Y.L. Colloidal and immobilized nanoparticles of lead xanthates. ACS Omega. 2019. Vol. 4(7). P. 11472-11480.

11. Mu Y., Li L., Peng Y. Surface properties of fractured and polished pyrite in relation to flotation. Minerals Engineering. 2017. Vol. 101(2). P. 10-19.

12. Асончик К.М., Требучкова Н.С., Аксенова Г.Я., Рябой В.И., Полькин В.Н. Разработка технологии обогащения медно-цинковой руды с получением медного концентрата высокого качества. Обогащение руд. 2009. № 1. C. 85-87. [Asonchik K.M., Trebuchkova N. S., Aksenova G. Ya., Ryaboy V.I., Polkin V.N. Development of a technology for beneficiation of copper-zinc ore to obtain high quality copper concentrate. Obogashchenie rud. 2009. No 1. P. 85-87. (In Russ.)]

13. Рябой В. И. Производство и применение флотационных реагентов в Российской Федерации Горный журнал. 2011. № 2. C. 17-20. [Ryaboy V. I. Production and usage of flotation reagents in Russia. Gornyi Zhurnal. 2011. 2. P. 17-20. (In Russ.)]

14. Сайкова Д.И., Чистяков Д.И., Сайкова С. В., Михлин Ю. Л., Кузьмин В. И. Поиск оптимальных условий синтеза и характеризация перспективного нанофлотореагента. Журнал Сибирского федерального университета. Химия. 2018. T. 11(1). C. 72-80. [Saikova D. I., Chistyakov D. I., Saikova S. V., Mikhlin Y.L., Kuzmin D. V. Synthesis and Characterization of Promising Nanosized Flotation Reagent. Journal of Siberian Federal University. Chemistry. 2018. Vol. 11(1). P. 72-80 (In Russ.)] 
15. Руководящий документ РД 52.24.382-2006. Массовая концентрация фосфатов и полифосфатов в водах. Методика выполнения измерений фотометрическим методом. Росгидромет, опубл. 2006. [RD52.24.382-2006. Mass concentration of phosphates and polyphosphates in waters. Photometric measurement technique. Rosgidromet, Publ. Date 2006. (In Russ.)]

16. Hergert W., Wriedt T. The Mie Theory. Basics and Applications. Springer Series in Optical Sciences. 2012. Vol. 169. P. 53-71.

17. Carius G. L. Ueber die äquivalente Ersetzung von Sauerstoff durch Schwefel. Justus Liebigs Annalen der Chemie. 1859. Vol. 112(2). P. 190-201.

18. Lee K., Kim H.-Y., Lotya M., Coleman J.N., Kim G.-T., Duesberg G.S., Electrical characteristics of molybdenum disulfide flakes produced by liquid exfoliation. Advanced Materials. 2011. Vol. 23(36). P. 4178-4182.

19. Cunningham G., Lotya M., Cucinotta C. S., Sanvito S., Bergin S. D., Menzel R., Shaffer M. S.P., Coleman J.N., Solvent exfoliation of transition metal dichalcogenides: dispersibility of exfoliated nanosheets varies only weakly between compounds. ACS Nano. 2012. Vol. 6(4). P. 3468-3480. 